RAINER WIMMER

\title{
Grenzen einer Normierung grammatischer Termini
}

\section{Einführende Bemerkungen}

Die Diskussion über die grammatische Terminologie im sprachunterricht allgemeinbildender schulen ist in den vergangenen Jahren vor allem dadurch erneut in Gang gekommen, dab die Kultusministerkonferenz 1982 einer Liste grammatischer Fachausdrücke zustimmte, die als orientierungshilfe und Richtschnur für den Grammatikunterricht dienen soll. Uber die Liste ist inzwischen ausgiebig diskutiert worden. Eine ganze Reihe von Diskussionsbeiträgen ist publiziert in dem von Albert Rasch 1983 herausgegebenen Band "Grammatische Terminologie". Es gibt darüber hinaus zahlreiche weitere Publikationen, und das nicht zu Unrecht, denn die mit der Termini-Liste erneut in besonders konturierter Form ins BewuBtsein gerückten Fragen der grammatischen Begrifflichkeit und deren Normierung im Hinblick auf den Anwendungsbereich Schule, sind ja keine Eintagsfliegen, sondern haben eine lange Tradition und werden auch in zukunft hoffentlich in der Diskussion bleiben - "hoffentlich" sage ich, denn solange die natürliche sprache sich wandelt, wird sich hoffentlich auch das sprechen über sprache als Teil des sprachwandels verändern und: Es wird hoffentlich auch weitere Fortschritte in der Erforschung der Sprache geben, so daß terminologische Veränderungen und die Reflexion über diese veränderungen unvermeidlich bleiben.

Für das Institut für deutsche Sprache habe ich auf der Grundlage von fruchtbaren Diskussionen in der Grammatikgruppe des Instituts und in den beiden Kommissionen für Rechtschreibfragen und für Sprachentwicklung Anfang 1983 eine stellungnahme $z u$ dem Termini-Katalog geschrieben, die in den Mitteilungen 9 des IDS publiziert ist (Wimmer 1983). An dieser stellungnahme wïrde ich auch heute in allen wesentlichen Punkten festhalten. 
In der IDS-Stellungnahme dominierte die fachwissenschaftliche Perspektive, d.h. die kritische würdigung der Termini-Liste war aus der sicht der sprach $w i s s$ e $n$ $c h$ a f $t$ erfolgt. Das wird auch im folgenden meine Perspektive sein, wenn ich einige Bemerkungen zur Normierbarkeit und zu den Grenzen der Normierung grammatischer Fachausdrücke mache. Ich möchte aber versuchen, diese Perspektive und den mit ihr verbundenen standpunkt in ein etwas anderes Licht zu rücken, als es bisher in der Kontroverse um die Termini-Normierung üblich war. Ich möchte das tun, indem ich nicht so sehr den Gegensatz zwischen fach w i s s e n $s \mathrm{ch}$ a $\mathrm{f} t \mathrm{i} \mathrm{c} h \mathrm{e} r$ sicht einerseits und fach $d \mathrm{i} d \mathrm{a} k$ $t$ i $s \mathrm{c} h \mathrm{e}$, unterrichtsmethodischer und pädagogischer sicht andererseits betone, sondern vielmehr die Gemeinsamkeiten und das gegenseitige Aufeinander-Angewiesen-Sein der Fachwissenschaft und der Fachdidaktik. Ich halte es für eines der bedauerlichsten Merkmale der bisherigen Diskussionen über den Termini-Katalog, daß bezüglich der Normierungsmöglichkeiten, -ziele und -grenzen ein allzu tiefer Graben zwischen fachwissenschaftlichen und fachdidaktischen bzw. unterrichtspraktischen Interessen gesehen worden ist. Insbesondere wurde in der Literatur festgestellt, daß von seiten der Fachdidaktiker und Bildungsexperten, die den Termini-Katalog vor allem vertreten, der Sprachwissenschaft und ihrer Entwicklung in der jüngsten Zeit ein zu starkes MiBtrauen entgegengebracht wird. Ferner: daB bei Fachdidaktikern und Bildungsexperten eine gewisse Theoriefeindlichkeit besteht. Dabei dürfte klar sein, daß man es im Ungang mit Fachausdrücken und speziell auch bei deren Normierung immer ganz wesentlich mit den Theorien zu tun hat, in denen die Termini ihre Basis haben und ohne die sie überhaupt nicht gebraucht, geschweige denn interpretiert werden können. selbstverständich sind mit der KMK-Termini-Liste ganz bestimmte theoretische Vorstellungen verbunden. Sonst wäre sie lediglich eine weitgehend inhaltsleere Nomenklatur. So kann Lewandowski beispielsweise feststellen: "Würde die KMK-Liste also verbindlich, muibte eine neue Grammatik für die deutsche schule geschrieben werden". 1 
Wenn ich dafür plädiere, die kluft zwischen Fachwissenschaft und Fachdidaktik nicht $z u$ tief $z u$ sehen, so möchte ich die spezifisch wissenschaftlichen Betrachtungsweisen, Interessen und Lebensformen einerseits und die fachdidaktischen und didaktischen andererseits keineswegs völlig nivellieren. Ich möchte der Fachdidaktik auch keine spezifisch fachwissenschaftliche Sehweise aufzwingen. Aber ich meine, daß Fachwissenschaft und Didaktik - was die Normierungsmöglichkeiten und -grenzen bei grammatischen Fachausdrücken angeht - eigentlich in einem Boot sitzen. Manchmal wird behauptet, das Interesse an und das Bedürfnis $z u$ einer Normierung und vereinheitlichung grammatischer Termini ergebe sich wesentlich erst aus dem didaktischen zusammenhang. Das Lernen und Lehren in der schule erfordere in ganz besonderem Maße Vereinheitlichung und Normierung im Dienste einer besseren Verständigung und besseren mnemotechnischen Leistung. Das trifft meines Erachtens in dieser einseitigen Pointierung nicht $z u$. Auch im wissenschaftlichen Bereich besteht im Interesse einer besseren Verständigung, im Interesse der Lehre und der Vermittlung und letztlich auch einer besseren Durchsetzbarkeit von Theorien ein starker zwang zur Normierung. Und jede Normierung - sei es im wissenschaftlichen oder im fachdidaktischen Bereich - unterliegt gewissen ähnlichen, allgemeinen Bedingungen, die einerseits von einem vernünftigen Normbegriff her diktiert werden und andererseits von den Möglichkeiten der Erkenntnis und Beschreibung des jeweils zur Debatte stehenden Gegenstands.

\section{Zur Kommunikation mit Termini}

Die wesentlichen Faktoren für eine Beurteilung der Grenzen einer Normierung grammatischer Termini ergeben sich meines Erachtens aus einer Analyse des Kommunikationstyps, der durch den Terminusgebrauch bestimmt wird. Termini sollen Verständigung ermöglichen und dadurch auch vermitteln zwischen a) einem theoriegesteuert erschlossenen Gegenstandsbereich, hier: der Grammatik, b) einem Terminusproduzenten bzw. -vertreter, der bestimmte Bedürfnisse und Interessen hat, seinen Terminus mit 
der von ihm festgelegten Bedeutung $z u$ gebrauchen und auch von anderen gebraucht zu erleben, und c) einem Terminusrezipienten bzw. -adressaten, der sich einerseits über den Terminusgebrauch den betreffenden Gegenstandsbereich erschließen möchte und der andererseits in dieser Gegenstandsorientierung mehr oder weniger freiwillig den Vorstellungen und Terminusgebrauchsbedingungen des Produzenten folgt. Die Grenzen für eine Normierung von Termini werden abgesteckt zwischen Terminusproduzenten, Terminusrezipienten und den Erfordernissen des Gegenstands, wobei diese Erfordernisse repräsentiert gedacht werden können durch all diejenigen, die als Wissenschaftler oder Fachdidaktiker oder sonst über sprache Nachdenkende bisher zur Erschliebung des Gegenstandsbereichs beigetragen haben. Wenigstens über die Erschliebung des Gegenstandsbereichs ergibt sich also eine kommunikationshistorische Perspektive: Kein Terminus kann auberhalb der Geschichte des bisherigen Denkens und Redens über den Gegenstand bestehen.

Die hier angedeuteten Relationen, die für den Kommunikationstyp bestimmend sind, lassen sich in einem schema gut im Uberblick darstellen. Das schema orientiert sich an den bekannten Darstellungen zu den Dimensionen der semiotik.

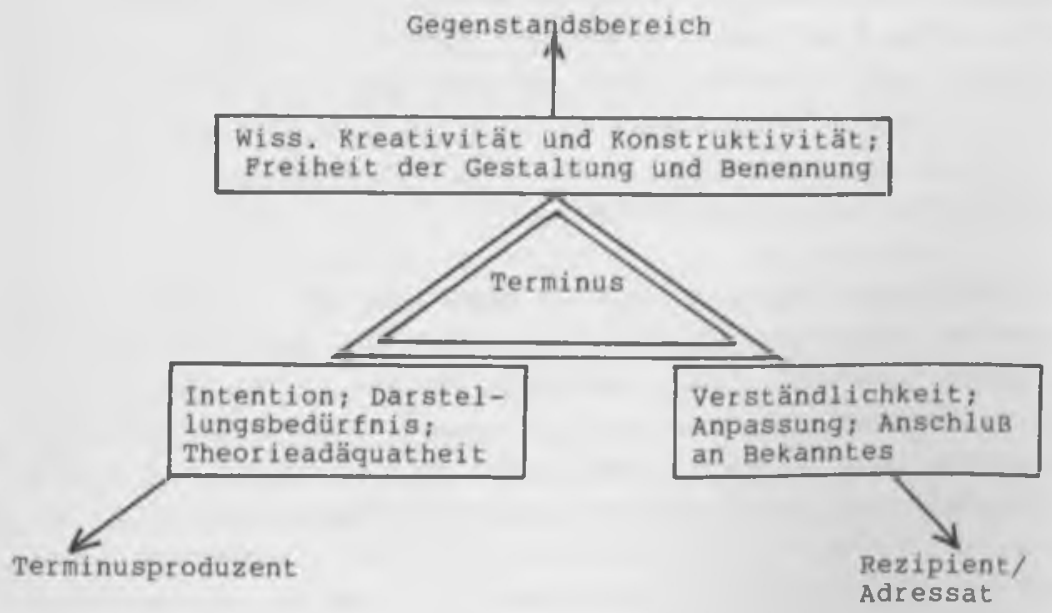


Im zentrum des schemas steht ein symbol für den Terminus. Von diesem symbol gehen pfeile aus auf "Gegenstandsbereich". "Terminusproduzent" und "Rezipient/Adressat", die pfeile deuten die Relationen an, in denen der Terminusgebrauch in fachsprachlich orientierten bzw. hier: grammatischen oder grammatiktheoretisch orientierten Kommunikationssituationen steht. Die Pfeile sind unterbrochen durch Kästen, in denen jeweils durch stichworte angedeutet ist, welche Bedeutung die Relationen für die Handlungsbeteiligten in den entsprechenden situationen im Hinblick auf unsere Fragestellung haben: Für den Terminusproduzenten bzw. für den positiv eingestellten Benutzer eines Terminus geht es darum, seine Intentionen und Darstellungsbedürfnisse im Hinblick auf den Gegenstand zur Geltung $z u$ bringen. Intentionen und Darstellungsbedürfnisse finden expliziten Ausdruck in der Theorie, die hinter dem Terminus steht. Von daher ergibt sich die Notwendigkeit, den Terminus jeweils theorieadäquat zu gebrauchen. - Ein Wissenschaftler und Terminusproduzent hat in seiner Arbeit der Konstituierung und Bearbeitung des Gegenstandsbereichs relative Gestaltungsfreiheiten. Diese Freiheiten beziehen sich auch auf die Benennung von Gegenständen. So hat der Ausdruck "Kreativität" seinen Platz in dem Kästchen zwischen "Terminus" und "Gegenstandsbereich". - Betrachtet man die Relation zwischen Terminus und Adressaten, so kommen vor allem Fragen der Verständlichkeit, der Anpassung an die Lebenswelt der Rezipienten und Möglichkeiten des Anschlusses an bereits vorhandenes Vorwissen der potentiellen Terminusbenutzer in den Blick.

Von den angedeuteten Relationen her, in denen der Terminus steht, lassen sich meines Erachtens kriterien für eine Bestimmung der Grenzen einer Normierung der Terminusbedeutung entwickeln. Darauf werde ich im folgenden unter Punkt 3 näher eingehen.

Die fachsprachliche Kommunikation wird wesentlich dadurch bestimmt, daß Termini explizit festgelegte, d.h. normierte Bedeutungen haben. Um ganz allgemeine Grenzen einer solchen Normierung einschätzen zu können, ist es notwendig, wenigstens eine 
grobe Begriffsunterscheidung zwischen "Regel", "Regelformulierung" und "Norm" vorzunehmen. ${ }^{2}$ Die meisten Regeln, die das Sprachhandeln der sprecher und Hörer tagtäglich leiten, werden blind befolgt. Sie sind den Sprachteilnehmern nicht bewust, und sie sind auch in Grammatiken nicht hinreichend beschrieben, d.h. es gibt für sie keine expliziten Regelformulierungen. Beispielsweise befolgen kompetente sprecher des Deutschen die komplizierten Regeln des deutschen substantivflexionssystems automatisch, ohne sich der Regel bewuBt zu sein. Die Regeln sind in gängigen Grammatiken auch kaum vollständig beschrieben, so daß der sprachteilnehmer auch keine Möglichkeit hat, sich die Regeln auf dem Wege über explizite Beschreibungen vor Augen zu führen. Normen unterscheiden sich dadurch von blind befolgten Regeln, daß sie a) explizite Formulierungen haben, die auf eine Normenquelle (Normierer) zurückgehen, und das sie b) aufgrund der expliziten Formulierungen geeignet sind, als Grundlage für Sanktionen gegenüber den Regelbenutzern zu dienen. Nur wenn eine grammatische Regel explizit formuliert ist, kann sie den Sprachteilnehmern vorgehalten werden, um auf ihre Einhaltung zu drängen oder eine Nicht-Befolgung zu sanktionieren.

Die hier angedeuteten Unterscheidungen sind für unseren zusammenhang insofern wichtig, als es zu erkennen gilt, das

- Normierungen (auch fachdidaktisch orientierte Normierungen) von grammatischen Termini nur über wissenschaftiche Regelformulierungen möglich sind;

- Normierungen von grammatischen Sachverhalten eine generelle Grenze darin finden, das sie blind befolgte Regeln des Sprachgebrauchs nicht tangieren können.

Die KMK-Liste grammatischer Termini zielt wohl darauf ab, weitreichend auf den sprachgebrauch einzuwirken. Sie kann solche Einwirkungen aber nur erreichen, wenn sie sich umfassend der wissenschaftlichen Erkenntnisse über die Grammatik des Deutschen versichert. 
3. Normierungsgrenzen aus den Kommunikationsbedingungen für Fachtermini

Ich komme auf die stichworte zurück, die in dem unter punkt 2 aufgefuhrten Schema die Relationen zwischen den Kommunikationsbeteiligten charakterisieren. Mit "Terminusproduzent" ist die Normierungsquelle angegeben. Der Normierer hat bestimmte Intentionen und Darstellungsbedürfnisse, die auch im Zusammenhang mit wissenschaftlichen Traditionen, Schulbildungen und Autoritäten zu sehen sind. Termini werden manchmal auch deswegen nicht akzeptiert, weil sie in ihren Bedeutungen Mitgemeintes mitführen, das wissenschaftlich und wissenschaftssozial bestimmt ist. Die KMK-Liste ist hier keineswegs neutral. So desavouiert sie beispielsweise den valenztheoretischen Ansatz in der syntax zugunsten traditioneller Vorstellungen von Satz und von Satzlehre. Sie tut das, obwohl der valenztheoretische Ansatz weithin (auch in der Duden-Grammatik) akzeptiert ist und nicht mit der vielgeschmähten sog. Linguistisierung des Deutschunterrichts in verbindung gebracht werden kann.

Die entscheidenden Normierungskriterien ergeben sich aus der Sicht des Terminusproduzenten durch die Theorie über die Grammatik, die er vertritt. Der Gebrauch von Termini verlangt die theoriebezogene Bestimmtheit der Gegenstände, die durch die Termini bezeichnet werden. Da die Gegenstände zu einem guten Teil durch den theoriebezogenen Gebrauch mitkonstituiert werden, gibt es über diese Bestimmtheit oft Kontroversen, gerade auch im wissenschaftlichen Bereich selbst. Was ist beispielsweise "Synonymie", was ist ein "Semem", was ein "enzyklopädisches semantisches Merkmal"? - Hier wird auch der Theoriewandel relevant: Wie hat sich etwa der Transformationsbegriff im Laufe der Geschichte der generativen Transformationsgrammatik seit 1957 gewandelt? oder: Wie ist er in der sprachdidaktik der 70er Jahre in der Bundesrepublik Deutschland verballhornt worden? - Was macht man, wenn das bein in sein Auto einmal als Artikel und einmal als possessivpronomen gelten soll? 
Solche Fragen kann der Terminusproduzent nur aufgrund von Theorien entscheiden, die er für vertretbar hält. Die Terminusbedeutungen ergeben sich für ihn aus den theoretischen zusammenhängen. Die Theorie (wobei Kohärenz, Widerspruchsfreiheit und Vollstänaigkeit eine wichtige Rolle spielen) definiert für ihn die Grenzen einer Normierung der Terminusbedeutung.

Zu der Relation zwischen Terminusproduzent und Gegenstandsbereich: Der Wissenschaftler, der zu bestimmten Terminusformulierungen kommt, muB auch eine gewisse kreativität und Freiheit in der Definition des Gegenstands und in der wahl der prädikationen, die er über den Gegenstand macht, für sich in Anspruch nehmen können. Nur so kann er neue Ideen in die Behandlung des Gegenstands einbringen; nur so kann er erfolgreich für den notwendigen Wandel von Terminologisierungen arbeiten. Unter den gegenwärtigen forschungs- und bildungspolitischen Bedingungen in der Bundesrepublik Deutschland haben an den Universitäten etablierte Wissenschaftler zweifellos gewisse Privilegien in der Wahrnehmung von Freiheiten bei der Definition von Gegenstandsbereichen. Mit diesen Privilegien und Rechten muB aber gerechnet werden. Die KMK-Liste grammatischer Termini nimmt darauf zu wenig Rücksicht. Sie mübte deutlich machen, inwieweit sie die von ihr vertretenen Normierungen heute für durchsetzbar hält.

Schlieblich $z$ der überaus wichtigen Relation von Terminusproduzent und Terminus zu den Rezipienten bzw. Adressaten. Hier spielt der Verständlichkeitsbegriff eine entscheidende Rolle ${ }^{3}$. Die Terminusnormierung muB sich an dem orientieren, was die Terminusrezipienten für wichtig, für integrierbar in ihren wissensbestand halten. Die KMK-Liste läßt hier wiederum sehr deutlich Einseitigkeiten erkennen: Sie kommt Eltern entgegen, insofern sie "traditionelle" Grammatiken favorisiert, was immer unter einer traditionellen Grammatik verstanden werden mag. Sie "dient" Lehrern, die im Laufe ihres studiums keine Grammatik "gelernt" haben. Sie ist ökonomisch für Verlage und für die Kultusbürokratie. 
Eigentlich müssen die "Bedürfnisse" der Rezipienten bei der Normierung und bei der Durchsetzung von Normen vorzüglich beachtet werden. Dies ist aber ein ganz weitgehend unbeackertes Feld. $2 u-$ mindest weis man kaum etwas darüber, wie und überhaupt in welchem MaBe Grammatiken von den Sprachteilhabern benutzt und tatsächlich in Anspruch genommen werden. Man weis kaum etwas darüber, welche Beschreibungssprache bei den potentiellen Grammatikbenutzern gut ankommt. Die verfasser der KMK-Liste haben sich über diese Frage anscheinend gar keine Gedanken gemacht. Es ist für sprachwissenschaftler und entsprechende Fachdidaktiker gleichermaßen erschreckend, daß die KMK einen Terminuskatalog verabschiedet, der die Rezipienten- bzw. Adressatenfrage so weitgehend ausklammert.

\section{Schlußbemerkungen}

Mein SchluBplädoyer geht dahin, bei Versuchen, die grammatische Terminologie für den schulunterricht nachhaltig und effektiv zu normieren, stärker den Normbegriff und die Grenzen einer Normierbarkeit von Termini zu reflektieren. Es müBte vielleicht auch möglich sein, etwas mehr Toleranz im Umgang mit terminologischen Festlegungen zu erreichen. Der Fachdidaktiker und Schulpraktiker müßte anerkennen, daß Termini von wissenschaftlern nicht willkürlich produziert werden, sondern auf der Grundlage von neuen Erkenntnissen und entsprechenden Theorien. Erkenntnisfortschritte führen $z u$ theoretischen Veränderungen, und diese führen zum wandel terminologischer systeme. Andererseits sollte der terminiproduzierende Wissenschaftler die Vermittlungsbedingungen für neue Erkenntnisse stärker berücksichtigen. Ubertriebene Terminologisierungen können sprachbarrieren aufbauen, die dem Erkenntnisfortschritt eher schaden als nützen. 


\section{Anmerkungen}

1 Lewandowski in Raasch 1983, S. 51-59.

2 Vgl. zum folgenden Kapitel 3 in Heringer u.a. 1977.

$3 \mathrm{Vgl}$. H.J. Heringer 1979.

\section{Literatur}

Ader, D. u.a. (1980): Nomen vs. Substantiv. Ein Votum für eine einheitliche Terminologie im Unterricht. In: Mitteilungen des Deutschen Germanistenverbandes, 27.3.1980, s. 32-36.

Beling, G./Wersig, G. (1979): Pragmatische Aspekte der Terminologienormung, in: Mentrup (Hrsg.) 1979, S. 144-154.

Cherubim, D. (1980): Grammatikographie. In: Althaus, H.P./Henne, H./Wiegand, H. E. (Hrsgg.), Lexikon der germanistischen Linguistik, 2. Auf1., Tübingen 1980 , S. 768-778.

DIN 2330 (1979): Begriffe und Benennungen, Berlin 1979.

DIN 2331 (1980): Begriffssysteme und ihre Darstellung, Berlin 1980.

DIN 2339 (1982): Ausarbeitung und Gestaltung von Veröffentlichungen mit terminologischen Feststellungen, Berlin 1982.

Felber, H. u.a. (Hrsgg.) (1979): Terminologie als angewandte Sprachwissenschaft. Gedenkschrift für Universitäts-prof. Dr. Eugen Wüster, München 1979.

Fleischer, w. (1973): Zur linguistischen Charakterisierung des Terminus in Natur- und Gesellschaftswissenschaften. In: Deutsch als Fremdsprache 4, 1973, S. 193-203.

Heringer, H.J. (1979): Verständlichkeit. Ein genuiner Forschungsbereich der Linguistik? In: ZGL 7, S. 255-278.

Heringer, H.J. u.a. (1977): Einfürung in die praktische semantik, Heidelberg 1977.

Heringer, H.J./Keller-Bauer (1984): Probleme einer gebrauchsgrammatischen Terminologie. In: SuL 53, 15. Jg., 1984, S. 65-86.

KMK-Liste (1982): Sekretariat der Ständigen Konferenz der Kultusminister der Länder in der Bundesrepublik Deutschland, Verzeichnis grundlegender grammatischer Fachausdrücke. Maschinenskript 1982.

Mentrup. w. (Hrsg.) (1979): Fachsprachen und Gemeinsprache. Jahrbuch 1978 des Instituts für deutsche Sprache, Düsseldorf 1979.

Raasch, A. (Hrsg.) (1983): Grammatische Terminologie (= forum Angewandte Linguistik, Bd. 1). Tübingen 1983.

Seibicke, w. (1982): Die Personennamen im Deutschen. Ber1in 1982.

Vogel,H. (1978): Das problem der grammatischen Terminologie in den Lehrplänen der Bundesländer. In: Der Deutschunterricht 30, 1978, s. 60-69.

Wimmer, R. (1983): IdS-stellungnahme zu dem "Verzeichnis grundlegender grammatischer Fachausdrücke (in der Kultugministerkonferenz zustimmend zur Kenntnis genommen am 26.2.1982)". In: Mitteilungen 9 des Instituts für deutsche Sprache, Mannheim 1983, S. 5-30.

wiegand, H. E. (1979): Definition und Terminologienormung, in: Felber u.a. (Hrsgg.) 1979, S. 101-148. 